\title{
From Multiple- to Single-Pulse All-Optical Helicity-Dependent Switching in Ferromagnetic Co/Pt Multilayers
}

\author{
G. Kichin, M. Hehn, J. Gorchon, G. Malinowski, J. Hohlfeld, and S. Mangin $\odot^{*}$ \\ Institut Jean Lamour, CNRS UMR 7198, Université de Lorraine, Vandouvre-lès-Nancy F-54506,
} France

(Received 25 January 2019; revised manuscript received 6 June 2019; published 9 August 2019)

\begin{abstract}
All-optical helicity-dependent switching in ferromagnetic $\mathrm{Co} / \mathrm{Pt}$ multilayers is investigated using magneto-optical microscopy and anomalous Hall effect measurements. A state diagram is built by studying the effect of pulse duration, fluence, and spot size. We use numerical solutions of the three-temperature model to explain that the all-optical helicity-dependent switching mechanism relies on the spin bath reaching temperatures close to the Curie point. Further insights into the reversal process are provided by the experimental demonstration of significant helicity-dependent reversal after a single laser pulse that reveals the involvement of direct angular momentum transfer. Moreover, based on the observation that longer pulse durations and larger spot sizes lead to enhanced reversal efficiency, we identify experimental conditions that lead to saturated magnetization reversal after just a few tens of laser pulses.
\end{abstract}

DOI: 10.1103/PhysRevApplied.12.024019

\section{INTRODUCTION}

All-optical switching (AOS) of the magnetization in metallic systems has been increasingly studied during the last decade since the discovery that a single femtosecond laser pulse could trigger the magnetization reversal in Gd-based ferrimagnetic alloys [1-3]. AOS offers the possibility of unprecedented fast and energy efficient magnetization manipulation and could impact applications in the fields of magnetic data storage, memory, computing, and logic. The technological potential has recently increased due to the discovery of AOS for a wide range of magnetic materials such as ferrimagnetic, synthetic antiferromagnetic, and ferromagnetic thin films and granular recording media [4,5]. However, all investigated materials other than Gd-based alloys and multilayers require a large number of laser pulses to achieve AOS and the magnetization within the reversed region is often not fully saturated.

Due to the complexity of laser pulse-induced magnetization dynamics, there exists no unified theory for AOS to date. Nevertheless, incomplete model descriptions have helped to identify the most important mechanisms at the origin of AOS for different material systems. In contrast to the initial belief that AOS is always driven by the transfer of angular momentum from the light field to the sample, two of the three most prominent model descriptions reveal temperature-induced magnetization dynamics as the driving force for AOS.

\footnotetext{
*stephane.mangin@univ-lorraine.fr
}

The first, applicable to Gd-based ferrimagnetic alloys and multilayers, identifies exchange scattering between antiferromagnetically coupled sublattices with significantly distinct demagnetization rates as the driving force for AOS via a transient ferromagnetic state [6,7]. Since this mechanism does not depend on the light helicity, it leads, in general, to toggle switching of present magnetic patterns for successive light pulses [8]. Nevertheless, deterministic all-optical helicity-dependent switching (AO HDS) can be achieved over a narrow fluence range that is governed by helicity-dependent absorption (magnetic circular dichroism, MCD) [9].

The second model interprets accumulative switching, observed in granular recording media after several hundreds of laser pulses, as the result of distinct superparamagnetic fluctuation rates for oppositely oriented grains that follow from MCD-dependent heating rates [10,11]. According to this model, single laser pulses with durations shorter than a few picoseconds, that is, shorter than the fastest superparamagnetic fluctuations, can never (even partially) reverse the magnetization of a fully saturated sample. Instead, they can randomize the magnetic orientations of the excited grains at best.

Only the third model attributes AO HDS to direct angular momentum transfer from the light to the sample (via the inverse Faraday effect) [12]. It equates AOS with thermomagnetic writing governed by optically induced magnetic field and temperature transients. Thus, its predictions rely on the interdependent results of three individual models for the calculation of (i) the strength and duration of the 
optically induced magnetic field, (ii) the electron- and lattice-temperature dynamics following the optical excitation of the sample, and (iii) the magnetization dynamics driven by the results of (i) and (ii).

Numeric simulations of the magnetization dynamics have shown that single laser pulses can cause fully saturated AO HDS of ferromagnetic materials provided the following conditions are met simultaneously $[12,13]$ : First, the temperature of the involved spins has to lie within a narrow band below the Curie point. It has to be high enough to enable linear (precessionless) magnetization reversal, but low enough to ensure that the impact of the optically induced magnetic field pulses is not surpassed by thermal fluctuations. Second, the duration and magnitude of the optically induced magnetic field pulses have to both exceed minimum values that are predominantly governed by the heating rate of the spins and by the field pulse duration, respectively.

According to the conditions above, saturated single shot AO HDS in ferromagnetic media relies on specially tuned field and temperature transients and can consequently only be achieved for particular sets of mutually dependent material and laser pulse parameters. The fact that even partial helicity-dependent reversal has not been observed in any single shot experiment on ferromagnetic media so far raises the question of whether those parameter sets exist at all. In other words, it might be impossible to find laser pulse parameters that, when combined with the material parameters of real ferromagnets, lead to single shot AO HDS. Indeed, recent theoretical work on the inverse Faraday effect suggests that optical excitations of ferromagnetic materials by 100 -fs laser pulses cannot create magnetic fields of sufficient strength and duration to drive single shot AO HDS (without heating the spins too much) [14]. However, the calculated fields are strong enough to explain measurements of multishot AO HDS in ferromagnetic films as the result of helicity-dependent domain expansion.

In this paper, we study the influence of laser pulse parameters such as pulse duration, beam size, fluency, and number of pulses in order to study AO HDS reversal in Ferromagnetic Co/Pt multilayers. From this study, we can build a state diagram, which highlights the conditions for which AO HDS is possible. Furthermore, we determine a set of parameters allowing reversal for a very low number of pulses. We even demonstrate a significant percentage of magnetization switching for a single pulse. Those results should not only have an impact on potential applications - energy efficient switching may be obtained - but they have strong implications for the fundamental understanding of the effect. Indeed, the single-pulse switching proves that angular transfer from the light to the magnetic material is key for AO HDS in Co/Pt. We show that all our results are in line with the third model introduced above that describes the reversal as the result of an angular momentum transfer or an inverse Faraday effect once the system receives enough energy to reach a certain spin temperature.

\section{AO HDS STATE DIAGRAM}

All the experiments are carried out on a glass/ $\mathrm{Ta}(3) /$ $\mathrm{Pt}(3) /[\mathrm{Pt}(0.7) / \mathrm{Co}(0.6)] / \mathrm{Pt}(3)$ stack (thicknesses in $\mathrm{nm}$ ) that will be referred to as $\mathrm{Co} / \mathrm{Pt}$. The sample shows a strong perpendicular magnetic anisotropy and a coercivity of $15 \mathrm{mT}$ at room temperature. The magnetic configuration of the full film is imaged with a MOKE microscope. A piece of the film is then patterned using optical lithography into a 5- $\mu \mathrm{m}$ wide Hall cross in order to electrically detect the magnetic state via the anomalous Hall effect (AHE) [3]. The 800-nm wavelength laser beam is kept at the same position during the excitation.

In order to find the optimal conditions for AO HDS, we systematically and independently vary three laser parameters: the fluence, the pulse duration, and the beam diameter. Figures 1(a)-1(c) show three characteristic MOKE images after exposure to $600 \sigma^{+}$laser pulses with a pulse duration of $1 \mathrm{ps}$ and for different laser fluences. As shown in Fig. 1(a), a fluence of $8.3 \mathrm{~mJ} / \mathrm{cm}^{2}$ leads to an alloptical fully switched magnetic domain (red) on an initially saturated film (blue). When increasing the fluence to $9.1 \mathrm{~mJ} / \mathrm{cm}^{2}$ [Fig. 1(b)], the area affected by the laser becomes larger while the central part gets demagnetized, which is consistent with previous works [5]. However, Fig. 1(c) shows that for an even larger fluence, the whole exposed area ends up in a multidomain state. This is rather unexpected under the common assumption that $\mathrm{AO}$ HDS happens within a fluence range. The absence of any reversed area at high fluences could be due to the following mechanisms. Increasing the average fluence reduces the spatial area within the fluence range, which could lead

(a)

(b)

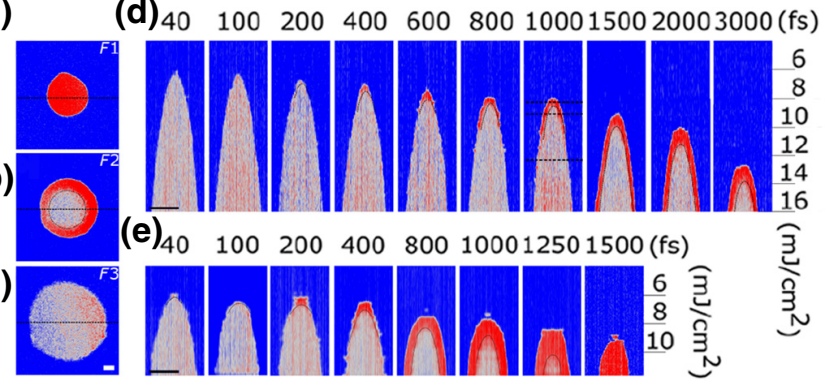

FIG. 1. (a)-(c) Magneto-optical Kerr images obtained after exposing the sample to 600 laser pulses with a pulse duration of $1 \mathrm{ps}$, a $\sigma^{+}$polarization, and laser fluences of $8.3 \mathrm{~mJ} / \mathrm{cm}^{2}$ (a), $9.1 \mathrm{~mJ} / \mathrm{cm}^{2}$ (b), and $12.3 \mathrm{~mJ} / \mathrm{cm}^{2}$ (c) corresponding to the lines in (d). The red and blue contrasts correspond to opposite perpendicular magnetization directions. (e)-(d) Cross section of the laser excited area for laser fluences between 4 and $18 \mathrm{~mJ} / \mathrm{cm}^{2}$ and various pulse durations for a laser spot diameter of $104 \mu \mathrm{m}$ (d) and $168 \mu \mathrm{m}$ (e). For (a)-(c), the white scale bar is $10 \mu \mathrm{m}$, for (d) and (e), the black bar is $50 \mu \mathrm{m}$. 
to a domain below the critical domain size. Alternatively, the excess heat in the center part of the Gaussian could diffuse outward thereby overheating and erasing the optically switched area. Due to the circular shape of the exposed area, we will now focus on their cross sections [black dotted lines in Figs. 1(a)-1(c)]. We record MOKE images for fluences between 4 and $20 \mathrm{~mJ} / \mathrm{cm}^{2}$; and pulse durations from $40 \mathrm{fs}$ to $3 \mathrm{ps}$, at a fixed helicity $\sigma^{+}$. We plot the resulting cross sections in Fig. 1(d) for a beam diameter of $104 \mu \mathrm{m}$ and in Fig. 1(e) for a beam diameter of $168 \mu \mathrm{m}$.

In the case of small diameter beams and pulses shorter than $200 \mathrm{fs}$, no switching can be observed in Fig. 1(d) independent of the fluence. However, for pulses with the same fluence and duration, switching is possible if the pulse diameter is increased [Fig. 1(e)]. Above 200 fs, the reversed area increases and a transition from a single domain state [Fig. 1(a)] to a mixed state occurs. In the mixed state, the center of the spot shows a multidomain state while the magnetization is fully reversed within an outer ring as previously reported. For a beam diameter of $168 \mu \mathrm{m}$, magnetization reversal can be obtained for laser pulses as short as $40 \mathrm{fs}$ [Fig. 1(e)]. Note that the spatial resolution of MOKE is about $1 \mu \mathrm{m}$ whereas the equilibrium domain size of the $\mathrm{Co} / \mathrm{Pt}$ sample is estimated to be around $100 \mu \mathrm{m}$ in Ref [15].

To verify our findings about the impact of pulse length and laser fluence on AOS derived from fluencedependent measurements at various fixed pulse lengths, we also perform complementary pulse length-dependent
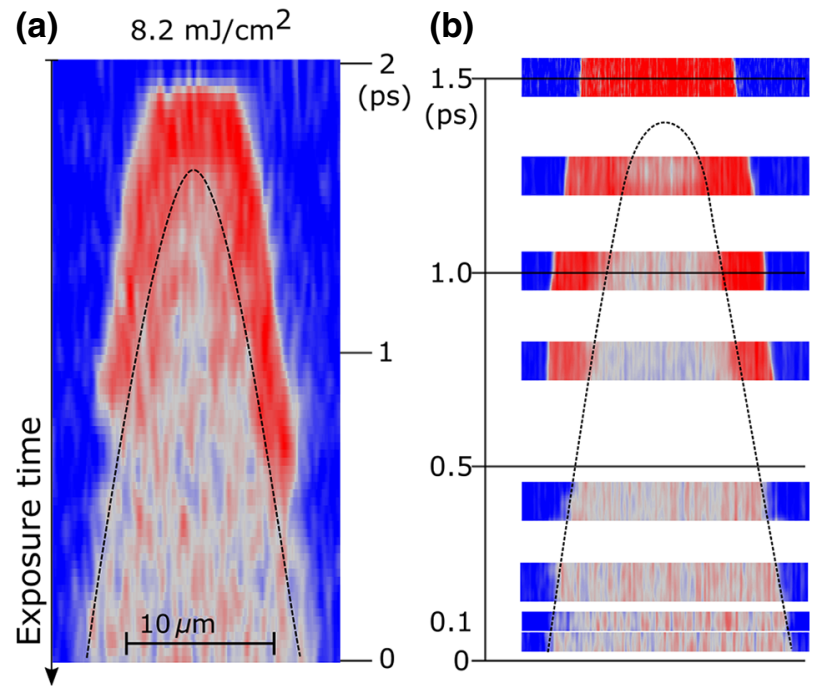

FIG. 2. (a) Cross section of the laser excited area as a function of time for a laser pulse duration tuned from $2 \mathrm{ps}$ to $40 \mathrm{fs}$. The laser helicity $(\sigma+)$, fluence $\left(8.2 \mathrm{~mJ} / \mathrm{cm}^{2}\right)$, and a beam diameter $(70 \mu \mathrm{m})$ are unchanged during the experiment. (b) Magnetization state map reconstructed from the data from the Fig. 1(e) for the laser fluence of $8.2 \mathrm{~mJ} / \mathrm{cm}^{2}$. Red color is used for magnetic domain state $\mathrm{M} \uparrow$ and blue for $\mathrm{M} \downarrow$. The dotted line defines the edge of the demagnetized region.

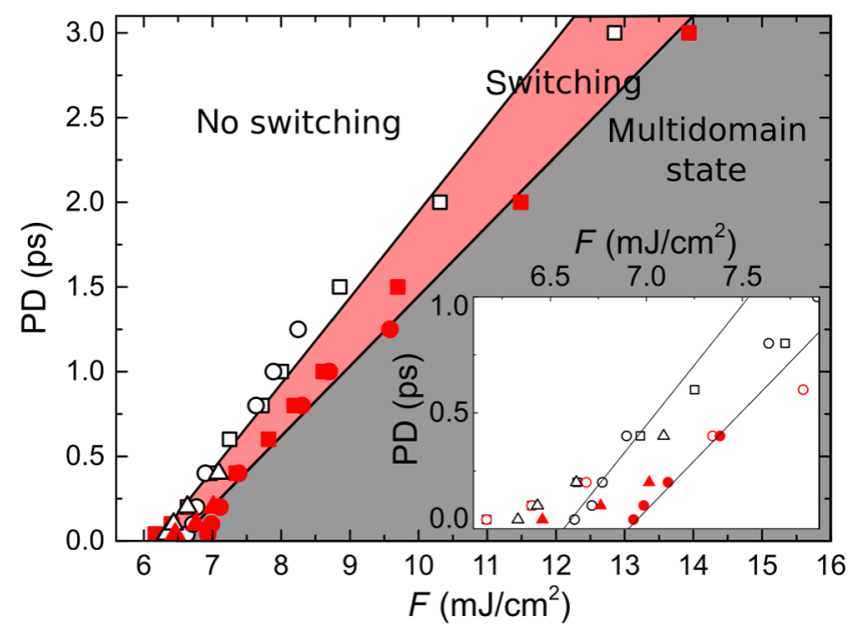

FIG. 3. Magnetization state diagram as a function of the laser fluence, $F$, and the pulse duration, PD, for a circularly polarized static beam of different diameters $(84 \mu \mathrm{m}$ for triangles, $104 \mu \mathrm{m}$ for square, and $168 \mu \mathrm{m}$ for dots) illuminating a Co/Pt. For a given fluence, the maximum (minimum) pulse duration for which we have AO HDS is plotted as filled (open) symbols. The inset shows a magnification of the diagram for the short pulse durations.

measurements at constant fluence. Figure 2(a) presents the evolution of the cross section of the laser excited region for a laser fluence of $8.2 \mathrm{~mJ} / \mathrm{cm}^{2}$ when the pulse duration is shortened from 2 ps to $40 \mathrm{fs}$. The excellent agreement with Fig. 2(b), depicting the corresponding results derived from Fig. 1, proves that a change in the pulse duration for a given laser fluence and a change in the laser fluence for a given pulse duration are equivalent.

The experimental data presented in Figs. 1 and 2 allowed us to construct the magnetization state diagram as a function of pulse duration and laser fluence as shown in Fig. 3. Three different areas can be defined: For low fluences and long pulses, the laser has no effect on the magnetic configuration. In the case of large fluences and short pulse durations, a multidomain state is observed. In between the two areas, for a certain range of pulse duration and laser fluence, full AO HDS corresponding to the reversal of a single magnetic domain is obtained. This state diagram reveals that for all pulse durations, a threshold fluence is required for AO HDS to be observed. Moreover, the fluence window for AO HDS widens for longer pulse durations, thereby indicating enhanced reversal efficiency for longer pulses. This observation is consistent with the previous reports on $\mathrm{Tb} / \mathrm{Co}[16]$ and $\mathrm{Co} / \mathrm{Pt}$ films [17].

\section{SWITCHING TEMPERATURE}

In order to explain our findings, we model the temperature dynamics of electron $\left(T_{e}\right)$, lattice $\left(T_{l}\right)$, and spin $\left(T_{s}\right)$ 
systems using the three-temperature model (3TM) [18]. We use the experimental pulse duration and associated fluence value for which switching is possible (Fig. 3).

For this, the spatial and temporal shapes of the laser pulse are described by the Gaussian

$$
I(x, t)=I_{0} \exp \left[-2 \ln 2\left(\frac{x^{2}}{x_{0}^{2}}+\frac{t^{2}}{\tau_{0}^{2}}\right)\right],
$$

where $I_{0}$ is the pulse amplitude intensity, $x_{0}$ is the beam diameter, and $\tau_{0}$ is the pulse duration [19-21]. Hence, the fluence is defined as $F(x)=I(x, 0) \tau_{0}$.

The time evolution is derived using a set of coupled differential equations given by

$$
\begin{aligned}
C_{e} \frac{d T_{e}}{d t} & =-G_{\mathrm{EL}}\left(T_{e}-T_{l}\right)-G_{\mathrm{ES}}\left(T_{e}-T_{s}\right)+P(t), \\
C_{l} \frac{d T_{l}}{d t} & =-G_{\mathrm{EL}}\left(T_{l}-T_{e}\right)-G_{\mathrm{SL}}\left(T_{l}-T_{s}\right), \\
C_{s} \frac{d T_{s}}{d t} & =-G_{\mathrm{ES}}\left(T_{s}-T_{e}\right)-G_{\mathrm{SL}}\left(T_{s}-T_{l}\right),
\end{aligned}
$$

where the electronic specific heat $\left(C_{e}\right)$ increases linearly with the electron temperature: $C_{e}=\gamma T_{e}$, and $C_{s}\left(C_{1}\right)$ is the spin (lattice) contribution to the specific heat. $G_{\mathrm{EL}}, G_{\mathrm{ES}}$, and $G_{\mathrm{SL}}$ are the electron lattice, electron spin, and spin lattice interaction constants that define the energy transfer rate from the electron system to the spin and the lattice. The values of the parameters such as the electron specific heat and coupling between the electron and lattice systems used are the ones given in Ref. [19]. The magnetic (lattice) specific heat and electron spin and spin lattice interactions are taken as free parameters. For the calculation, we consider that the spin lattice interaction time is longer than the spin electron interaction time and the electron-electron interaction is shorter than the spin-spin and lattice-lattice as in Ref. [22]. All the parameters used are shown in Table I.

Numerical solutions of the three-temperature model show (see Fig. 4) that neither the electronic peak temperature nor the final phonon's temperature remain constant for different pulse durations and fluences leading to $\mathrm{AO}$ HDS. However, the maximum spin temperature seems

TABLE I. Values of the parameters used in the three temperature model simulations.

\begin{tabular}{ll}
\hline \hline & $\mathrm{Co} / \mathrm{Pt}$ \\
\hline$\gamma\left(\mathrm{J} \mathrm{m}^{-3} \mathrm{~K}^{-2}\right)$ & 720 \\
$C_{1}\left(10^{6} \mathrm{~J} \mathrm{~m}^{-3} \mathrm{~K}^{-1}\right)$ & 3 \\
$C_{S}\left(10^{6} \mathrm{~J} \mathrm{~m}^{-3} \mathrm{~K}^{-1}\right)$ & 0.3 \\
$G_{\mathrm{el}}\left(10^{16} \mathrm{~W} \mathrm{~m}^{-3} \mathrm{~K}^{-1}\right)$ & 264 \\
$G_{\mathrm{es}}\left(10^{16} \mathrm{~W} \mathrm{~m}^{-3} \mathrm{~K}^{-1}\right)$ & 100 \\
$G_{\mathrm{sl}}\left(10^{16} \mathrm{~W} \mathrm{~m}^{-3} \mathrm{~K}^{-1}\right)$ & 0.1 \\
\hline \hline
\end{tabular}

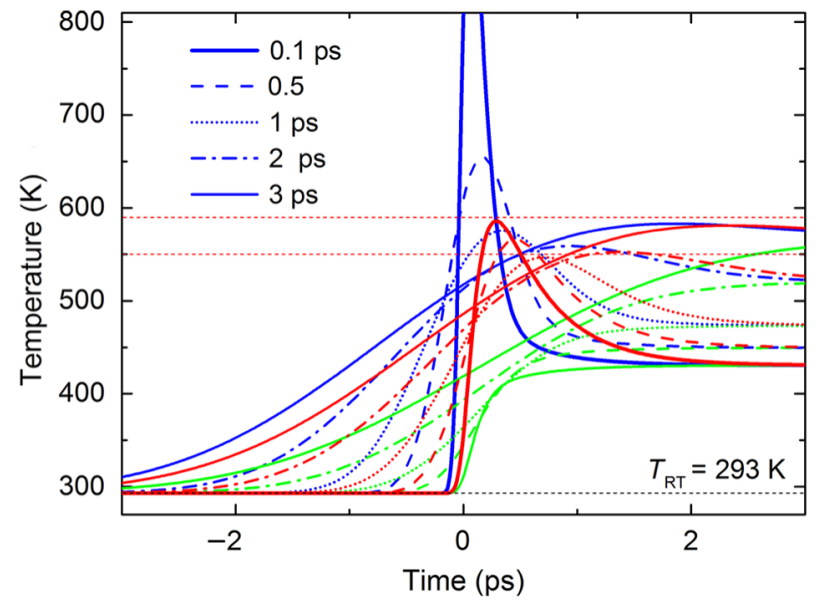

FIG. 4. Evolution of the temperatures of electrons (blue), lattice (green), and spin (red) as a function of time for five different pulses duration ( $0.1 \mathrm{ps}, 0.5 \mathrm{ps}, 1 \mathrm{ps}, 2 \mathrm{ps}$, and $3 \mathrm{ps})$. The maximum intensity of the laser pulse definition origin of time. The values of the fluence for a pulse duration are taken from the experiment shown in Fig. 1. The evolutions, starting from room temperature, $T_{\mathrm{RT}}$, are obtained using the $3 \mathrm{TM}$ equations (2) and parameters from Table I.

to be within a certain temperature range. Moreover, this temperature range is close to the $\mathrm{Co} / \mathrm{Pt}$ Curie temperature. The agreement of this finding with the respective spin temperature-related switching criterion suggests that AO HDS in $\mathrm{Co} / \mathrm{Pt}$ is the result of thermomagnetic writing by optically induced fields. Moreover, solutions of the three-temperature model for fixed fluences reveal that the maximum spin temperature decreases with increasing pulse length. Thus, the disappearance of AO HDS for pulses longer than the fluence-dependent upper limit identified in our state diagram can be understood as a consequence of insufficient spin heating.

To corroborate our claim that AO HDS in $\mathrm{Co} / \mathrm{Pt}$ indeed satisfies the spin temperature-related switching criterion, that is, that the spin temperature is very close to the Curie point during the reversal, we present the evolution of the magnetic configuration as a function of the laser fluence as measured in the presence of a small perpendicularly applied magnetic field in Fig. 5. The sample initially magnetized $\mathrm{M} \downarrow$ is irradiated with a laser of $\sigma^{+}$ helicity. AO HDS is observed for a $40-\mathrm{fs}$ pulse duration. An external magnetic field is applied perpendicular to the sample. The magnitude of the magnetic field $H_{\text {ext }}$ is much smaller than the coercive field at a room temperature $H_{C}=150 \mathrm{Oe}$. A positive (negative) external field helps (suppresses) the magnetization reversal. Even for a field of 1 Oe or -1 Oe, the area affected by the switching changes. The area slightly increases for 1 Oe and slightly decreases for -1 Oe, whereas a 10-Oe field significantly increases the size of the switched area and decreases the demagnetized area. For -10 Oe, AO HDS is no longer 


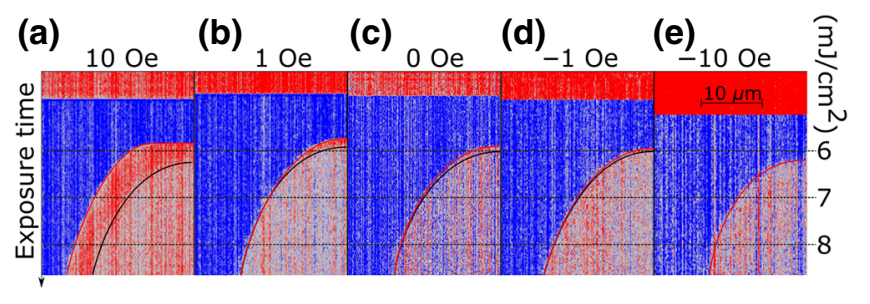

FIG. 5. Magneto-optical contrast in a cross section of the $\mathrm{Co} / \mathrm{Pt}$ film under the repetitive irradiation of the pump pulses as a function of the laser power, obtained for various external magnetic fields. Laser excitation has a fixed $\sigma^{+}$helicity, pulse duration of $40 \mathrm{fs}$, laser power of $0.75 \mathrm{~mW}$, and a $5-\mathrm{kHz}$ repetition rate. The sample is initially saturated in a large positive magnetic field $(\mathrm{M} \uparrow)$ and then negative field (M $\downarrow)$ in order to normalize the signal [red for magnetization up (M $\uparrow$ ) and blue for magnetization down $(\mathrm{M} \downarrow)]$.

observed. The strong effect of such small fields indicates that the system is close to its ordering temperature.

\section{TOWARD SINGLE-PULSE SWITCHING}

Having identified thermomagnetic writing by optically induced fields as the reversal mechanism of AO HDS in $\mathrm{Co} / \mathrm{Pt}$, we now turn our attention to the key question of whether optimized laser parameters can lead to switching after a single pulse. Again, up until now, all the reported works have shown that a large number of pulses is required to observe AO HDS in uniformly magnetized samples that do not contain Gd. El Hadri et al. have shown that a few hundred ultrashort pulses are required for switching $\mathrm{Co} / \mathrm{Pt}$ or $\mathrm{Co} / \mathrm{Tb}$ alloys [23]. By fine tuning of the excitation parameters, we are able to drastically reduce the number of pulses required to obtain HD AOS. Figure 6 shows that full AO HDS can be reached after 50 pulses of 2-ps duration. Even 10 pulses under the same condition are enough to reverse the magnetization even though the reversal is not as uniform.

In order to enable studies on the effect of a single laser pulse, the $\mathrm{Co} / \mathrm{Pt}$ film is patterned in a 5- $\mu \mathrm{m}$ Hall cross and the out-of-plane component of the magnetization is monitored via the anomalous Hall voltage (AHV) [24]. The

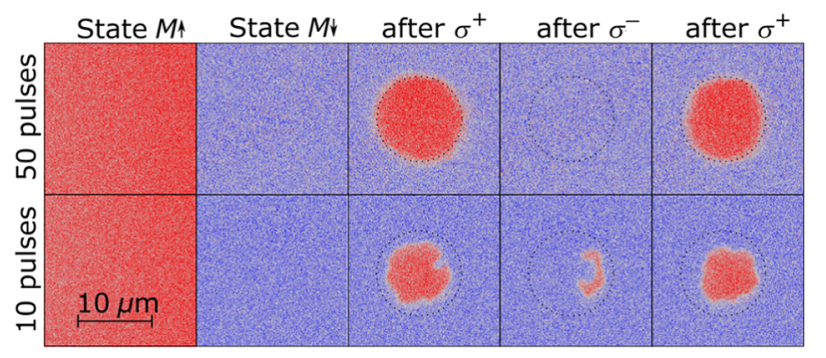

FIG. 6. Magneto-optical images obtained after 50 (top) and 10 (bottom) using a 2-ps laser pulse and a fluence of $11 \mathrm{~mJ} / \mathrm{cm}^{2}$.
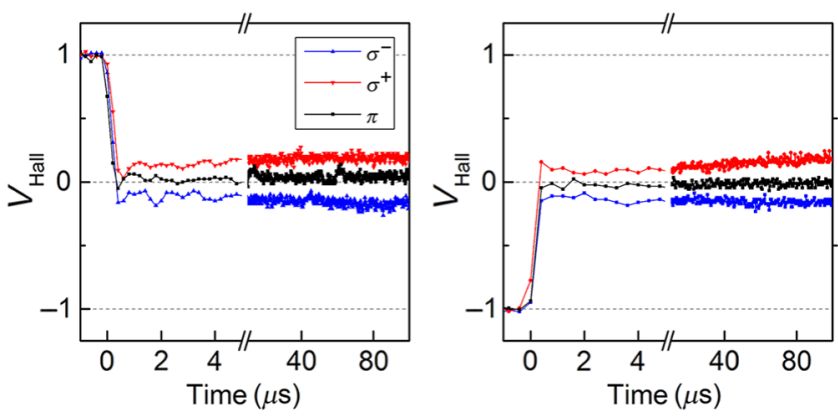

FIG. 7. Normalized Hall voltage $V_{\mathrm{AHV}}$ showing the timeresolved magnetization dynamics of $\mathrm{Co} / \mathrm{Pt}$. The signal is normalized to the values measured for saturation of magnetization. The sample with initially saturated magnetization is excited with a single laser pulse of helicity $\sigma^{+}$(red), $\sigma^{-}$(blue), and linear $\pi$ (black). The two graphs correspond to opposite initial magnetization directions. Switching time is estimated as less than $200 \mathrm{~ns}$. Pulse duration is approximately equal to 2 ps. Laser fluence is approximately equal to $11 \mathrm{~mJ} / \mathrm{cm}^{2}$.

center of the Hall cross is excited with single 2-ps laser pulses with a fluence of $11 \mathrm{~mJ} / \mathrm{cm}^{2}$ for different circular and linear polarizations. The diameter of the beam $(70 \mu \mathrm{m})$ is larger than the 5- $\mu \mathrm{m}$ cross width.

As shown in Fig. 7, exciting the sample with a linear polarization leads to $V_{\mathrm{AHV}}=0$, which is characteristic of a multidomain state, whereas circularly polarized laser pulses induce a signal $V_{\mathrm{AHV}}= \pm 0.1$ whose sign is governed by the polarization of the light. The transition between the initial and final states takes less than $200 \mathrm{~ns}$, which is the time resolution of our setup. Since our measurements are performed on uniformly magnetized samples, the observation of single-pulse AO HDS cannot be explained by magnetic circular dichroism (as is done in Refs [25] and [26]). Thus, our results prove that AO HDS in $\mathrm{Co} / \mathrm{Pt}$ is driven by angular momentum transfer from the light to the magnetic material.

\section{CONCLUSION}

We study the influence of laser pulse parameters such as pulse duration, beam size, fluence, and number of pulses on magnetization switching and demagnetization in Ferromagnetic $\mathrm{Co} / \mathrm{Pt}$ multilayers. From this study, we build a state diagram showing the magnetization state of the ferromagnetic thin film after the laser exposure as a function of the laser fluence and the pulse duration. We optimize the laser pulse characteristics to observe full magnetization switching after 50 pulses and significant switching after only 10 pulses. We then perform a single laser pulse experiment showing that it is possible to obtain $10 \%$ switching for an optimized laser pulse. Those experimental results allow us conclude that magnetic circular dichroism alone cannot explain AO HDS. Combining the experimental results and the three-temperature 
model suggests that the criteria to observe switching is that the laser brings enough heat so that the spin temperature can reach a value close to the spin-ordering temperature. At this temperature, we expect that a moderate angular momentum transfer from the circular polarized light to the magnetic material should be sufficient to generate the switching. This conclusion is in agreement with the theoretical prediction provided by Oppenner et al. $[27,28]$.

\section{ACKNOWLEDGMENTS}

This work was supported by the ANR-NSF Project, Grant No. ANR-13-IS04-0008-01, COMAG, Grant No. ANR-15-CE24-0009, UMAMI, and by the ANR-Labcom Project LSTNM, by the Institut Carnot ICEEL for the project "Optic-switch" and Matelas, and by the French PIA project "Lorraine Université d'Excellence," Grant No. ANR-15IDEX-04-LUE. Experiments were performed using equipment from the TUBE. Davm was funded by FEDER (EU), ANR, Région Grand Est, and Métropole Grand Nancy.

[1] C. D. Stanciu, F. Hansteen, A. V. Kimel, A. Kirilyuk, A. Tsukamoto, A. Itoh, and T. Rasing, All-Optical Magnetic Recording with Circularly Polarized Light, Phys. Rev. Lett. 99, 047601 (2007).

[2] A. Kirilyuk, A. V. Kimel, and T. Rasing, Laser-induced magnetization dynamics and reversal in ferrimagnetic alloys, Rep. Prog. Phys. 76, 026501 (2013).

[3] M. S. El Hadri, M. Hehn, G. Malinowski, and S. Mangin, Materials and devices for all-optical helicity-dependent switching, J. Phys. D Appl. Phys. 50, 133002 (2017).

[4] S. Mangin, M. Gottwald, C. Lambert, D. Steil, V. Uhlír, L. Pang, M. Hehn, S. Alebrand, M. Cinchetti, G. Malinowski, Y. Fainman, M. Aeschlimann, and E. E. Fullerton, Engineered materials for all-optical helicity-dependent magnetic switching, Nat. Mater. 13, 286 (2014).

[5] C.-H. Lambert, S. Mangin, B. S. D. C. S. Varaprasad, Y. K. Takahashi, M. Hehn, M. Cinchetti, G. Malinowski, K. Hono, Y. Fainman, M. Aeschlimann, and E. E. Fullerton, All-optical control of ferromagnetic thin films and nanostructures, Science 345, 1337 (2014).

[6] I. Radu, K. Vahaplar, C. Stamm, T. Kachel, N. Pontius, H. A. Dürr, T. A. Ostler, J. Barker, R. F. L. Evans, R. W. Chantrell, A. Tsukamoto, A. Itoh, A. Kirilyuk, T. Rasing, and A. V. Kimel, Transient ferromagnetic-like state mediating ultrafast reversal of antiferromagnetically coupled spins, Nature 472, 205 (2011).

[7] A. J. Schellekens and B. Koopmans, Microscopic model for ultrafast magnetization dynamics of multisublattice magnets, Phys. Rev. B 87, 020407 (2013).

[8] T. A. Ostler, et al, Ultrafast heating as a sufficient stimulus for magnetization reversal in a ferrimagnet, Nat. Commun. 3, 666 (2012).
[9] A. R. Khorsand, M. Savoini, A. Kirilyuk, A. V. Kimel, A. Tsukamoto, A. Itoh, and Th Rasing, Role of Magnetic Circular Dichroism in All-Optical Magnetic Recording, Phys. Rev. Lett. 108, 127205 (2012).

[10] J. Gorchon, Y. Yang, and J. Bokor, Model for multishot allthermal all-optical switching in ferromagnets, Phys. Rev. B 94, 020409(R) (2016).

[11] M. O. A. Ellis, E. E. Fullerton, and R. W. Chantrell, All-optical switching in granular ferromagnets caused by magnetic circular dichroism, Sci. Rep. 6, 1 (2016).

[12] K. Vahaplar, A. M. Kalashnikova, A. V. Kimel, D. Hinzke, U. Nowak, R. Chantrell, A. Tsukamoto, A. Itoh, A. Kirilyuk, and T. Rasing, Ultrafast Path for Optical Magnetization Reversal via a Strongly Nonequilibrium State, Phys. Rev. Lett. 103, 117201 (2012).

[13] R. W. Chantrell, private communications

[14] A. Kundu and S. Zhang, Effect of laser induced orbital momentum on magnetization switching, J. Magn Magn Mater. 454, 165 (2018).

[15] M. S. El Hadri, M. Hehn, P. Pirro, C. H. Lambert, G. Malinowski, E. E. Fullerton, and S. Mangin, Domain size criterion for the observation of all-optical helicity-dependent switching in magnetic thin films, Phys. Rev. B. 94, 064419 (2016).

[16] S. Alebrand, M. Gottwald, M. Hehn, D. Steil, M. Cinchetti, D. Lacour, E. E. Fullerton, M. Aeschlimann, and S. Mangin, Light-induced magnetization reversal of highanisotropy TbCo alloy films, Appl. Phys. Lett. 101, 162408 (2012).

[17] R. Medapalli, D. Afanasiev, D. K. Kim, Y. Quessab, S. Manna, S. A. Montoya, A. Kirilyuk, T. Rasing, A. V. Kimel, and E. E. Fullerton, Multiscale dynamics of helicity-dependent all-optical magnetization reversal in ferromagnetic Co/Pt multilayers, Phys. Rev. B 96, 224421 (2017).

[18] E. Beaurepaire, J.-C. Merle, A. Daunois, and J.-Y. Bigot, Ultrafast Spin Dynamics in Ferromagnetic Nickel, Phys. Rev. Lett. 76, 4250 (1996).

[19] N. Bergeard, M. Hehn, S. Mangin, G. Lengaigne, F. Montaigne, M. L. M. Lalieu, B. Koopmans, and G. Malinowski, Hot-Electron-Induced Ultrafast Demagnetization in $\mathrm{Co} / \mathrm{Pt}$ Multilayers, Phys. Rev. Lett. 117, 147203 (2016).

[20] J. Mendil, P. Nieves, O. Chubykalo-Fesenko, J. Walowski, T. Santos, S. Pisana, and M. Münzenberg, Resolving the role of femtosecond heated electrons in ultrafast spin dynamics, Sci. Rep. 4, 3980 (2014).

[21] K. C. Kuiper, T. Roth, A. J. Schellekens, O. Schmitt, B. Koopmans, M. Cinchetti, and M. Aeschlimann, Spinorbit enhanced demagnetization rate in co/pt-multilayers, Appl. Phys. Lett. 105, 202402 (2014).

[22] A. Kirilyuk, A. V. Kimel, and Th. Rasing, Ultrafast optical manipulation of magnetic order, Rev. Mod. Phys. 82, 2731 (2010).

[23] M. S. El Hadri, P. Pirro, C. Lambert, Y. Quessab, M. Hehn, F. Montaigne, G. Malinowski, and S. Mangin, Two types of all-optical magnetization switching mechanisms using femtosecond laser pulses, Phys. Rev. B 94, 064412 (2016).

[24] M. S. El Hadri, P. Pirro, C. Lambert, N. Bergeard, M. Hehn, G. Malinowski, F. Montaigne, R. Medapalli, E. E. Fullerton, S. Mangin, M. S. El Hadri, P. Pirro, 
C. Lambert, N. Bergeard, and M. Hehn, Electrical characterization of all-optical helicity-dependent switching in ferromagnetic Hall crosses, Appl. Phys. Lett. 108, 092405 (2016).

[25] Y. Tsema, G. Kichin, O. Hellwig, V. Mehta, A. V. Kimel, A. Kirilyuk, and T. Rasing, Helicity and field dependent magnetization dynamics of ferromagnetic $\mathrm{Co} / \mathrm{Pt}$ multilayers, Appl. Phys. Lett. 109, 072405 (2016).

[26] Y. Quessab, R. Medapalli, M. S. El Hadri, M. Hehn, G. Malinowski, E. E. Fullerton, and S. Mangin, Helicity-dependent all-optical domain wall motion in ferromagnetic thin films, Phys. Rev. B 97, 054419 (2018).

[27] M. Berritta, R. Mondal, K. Carva, and P. M. Oppeneer, $A b$ Initio Theory of Coherent Laser-Induced Magnetization in Metals, Phys. Rev. Lett. 117, 137203 (2016).

[28] R. John, M. Berritta, D. Hinzke, C. Müller, T. Santos, H. Ulrichs, P. Nieves, J. Walowski, R. Mondal, O. Chubykalo-Fesenko, J. McCord, P. M. Oppeneer, U. Nowak, and M. Münzenberg, Magnetisation switching of FePt nanoparticle recording medium by femtosecond laser pulses, Sci. Rep. 7, 4114 (2017). 